\title{
L'ablation des polymères par irradiation laser excimère : le processus physique et la structure du polymère
}

\author{
D. Martinez, Cl. Dicara, Fr. Hanus, K. Kolev et L.D. Laude \\ Université de Mons-Hainaut, 23 avenue Maistriau, 7000 Mons, Belgique
}

\begin{abstract}
Résumé : L'ablation des polymères est un phénomène mis en œuvre en irradiant ces matériaux avec une pulsation de lumière produite par une source laser excimère. Ce phénomène se manifeste par une émission de matière à partir d'un seuil de densité d'énergie superficielle ou fluence. Au-delà de ce seuil, la quantité de matière émise par pulsation croît d'abord linéairement avec la fluence, puis tend à se stabiliser à une valeur maximum. Ces caractéristiques sont ici mises en évidence pour trois polymères choisis pour, d'une part leur parenté structurelle et d'autre-part, leur tenue élevée en température : le polyéthersulfone PES, le polyéthylèneteréphtalate PET et le polysulfure de phénylène PPS. L'analyse qui est ici proposée de ces caractéristiques met l'accent sur deux aspects importants, à savoir le sens physique du seuil d'émission et la dynamique même de l'émission. On montre en particulier qu'en tenant compte de la pénétration du rayonnement, la valeur du seuil (en $\mathrm{J} / \mathrm{cm}^{3}$ ) est directement proportionnée à la taille du monomère, marquant ainsi la linite de stabilité morphologique de la chaîne polymérique en regard d'un apport d'énergie lumineuse.
\end{abstract}

\section{INTRODUCTION}

L'ablation est un phénomène d'émission de matière qui peut être induit par irradiation d'une surface au moyen du faisceau de lumière UV émis en régime pulsé par une source laser excimère. Elle a fait l'objet d'un nombre considérable de travaux depuis son initiation en 1985 [1] et a été, depuis cette date, mise en œuvre en particulier sur des matériaux polymériques présentant un intérêt pratique [2]. Parallèlement, l'interprétation des caractéristiques de l'ablation, presque uniquement en terme de taux d'ablation en fonction de la densité d'énergie superficielle (ou "fluence" en $\mathrm{J} / \mathrm{cm}^{2}$ ) du faisceau laser à son impact sur le matériau cible, a été abordée très généralement par des modèles mathématiques paramétrisés basés sur la notion de transfert instantané au réseau de l'énergie optique absorbée, sous forme d'un front de chaleur diffusant à partir de la surface irradiée vers le volume du matériau [3]. De ce point de vue, une description cinétique du phénomène a toujours été privilégiée, sous forme d'un bilan d'énergie globalisé et sans relation avec la nature du matériau (atomes, liaisons interatomiques), au détriment de la dynamique propre du phénomène. C'est précisément la dynamique de l'ablation que se propose d'aborder cette présentation, au travers d'une description physique de son mécanisme.

Deux aspects essentiels du phénomène de l'ablation ont semble-t-il été constamment écartés de l'analyse: d'une part qu'il s'agissait toujours à l'origine d'une interaction "optique" entre lumière et matière générant en premier lieu des électrons de conduction (et donc des atomes momentanément ionisés) et secondairement des états vibratoires, et d'autre part que l'émission de lumière par une source excimère n'est pas instantanée mais perdure pendant 30 à $40 \mathrm{nsec}$. De fait, l'irradiation se développe sur une période de temps qui se trouve être comparable, dans les diélectriques, à la durée de vie des électrons de conduction générés lors de l'absorption de photons. C'est l'incidence de ces deux particularités qui permet de proposer un mécanisme rationnel de l'ablation et contribue à définir le sens physique du seuil d'ablation dans les polymères. 


\section{MATERIAUX ET RESULTATS EXPERIMENTAUX}

Dans ce travail, le phénomene de l'ablation a été étudié pour trois polymères choisis pour leur parenté structurelle et leur tenue élevée en température: le polyéthersulfone PES $\left(255^{\circ} \mathrm{C}\right)$, le polyéthylèneteréphtalate PET $\left(226^{\circ} \mathrm{C}\right)$ et le polysulfure de phénylène PPS $\left(288^{\circ} \mathrm{C}\right)$. Les nombres d'électrons de valence par monomère sont respectivement de 72,80 et 34 . On note la faible population électronique du monomère PPS par rapport aux deux autres monomères, alors que le polymère correspondant possède nettement la meilleure tenue thermique.

Les irradiations ont été réalisées à $248 \mathrm{~nm}$ et à cette longueur d'onde, la réflectivité a été respectivement mesurée a 0,07 (PES), 0,09 (PET) et 0,08 (PPS). A chaque fluence testée $E$ $\left(\mathrm{en} \mathrm{J} / \mathrm{cm}^{2}\right.$ ) et sur chaque polymère, une série de 20 irradiations successives a été réalisée à 1 sec d'intervalle et la profondeur de matière émise a été mesurée au profilomètre avant d'être réduite à la profondeur $\Delta z$ de matière émise par irradiation. Des séries comparatives de 10 irradiations successives ont été aussi réalisées qui ont confirmé ces valeurs de $\Delta z$. Les graphes rapportant les valeurs de $\Delta z$ obtenues en fonction de la fluence $E$ pour les trois polymères sont représentés Fig. I. Trois quantités sont définies pour chaque polymère: la fluence seuil $E_{0}$ (en $\left.\mathrm{J} / \mathrm{cm}^{2}\right)$, la "pente" $\chi\left(\mathrm{en} \mathrm{cm}^{3} / \mathrm{J}\right)$ ou accroissement de la profondeur ablaté par irradiation, que l'on observe être quasi-linéaire à partir du seuil et un peu au-delà, et $z_{0}$ (en $\mu \mathrm{m}$ ) la valeur asymptotique de la profondeur ablatée maximum, déterminée approximativement sur graphe Si l'ablation (par $\Delta z$ ) ne se manifeste pas de façon mesurable en dessous du seuil, on remarque le développement d'une certaine rugosité sur la surface irradiée à mesure qu'on accrô̂t $E$ pour se rapprocher de $E_{0}$
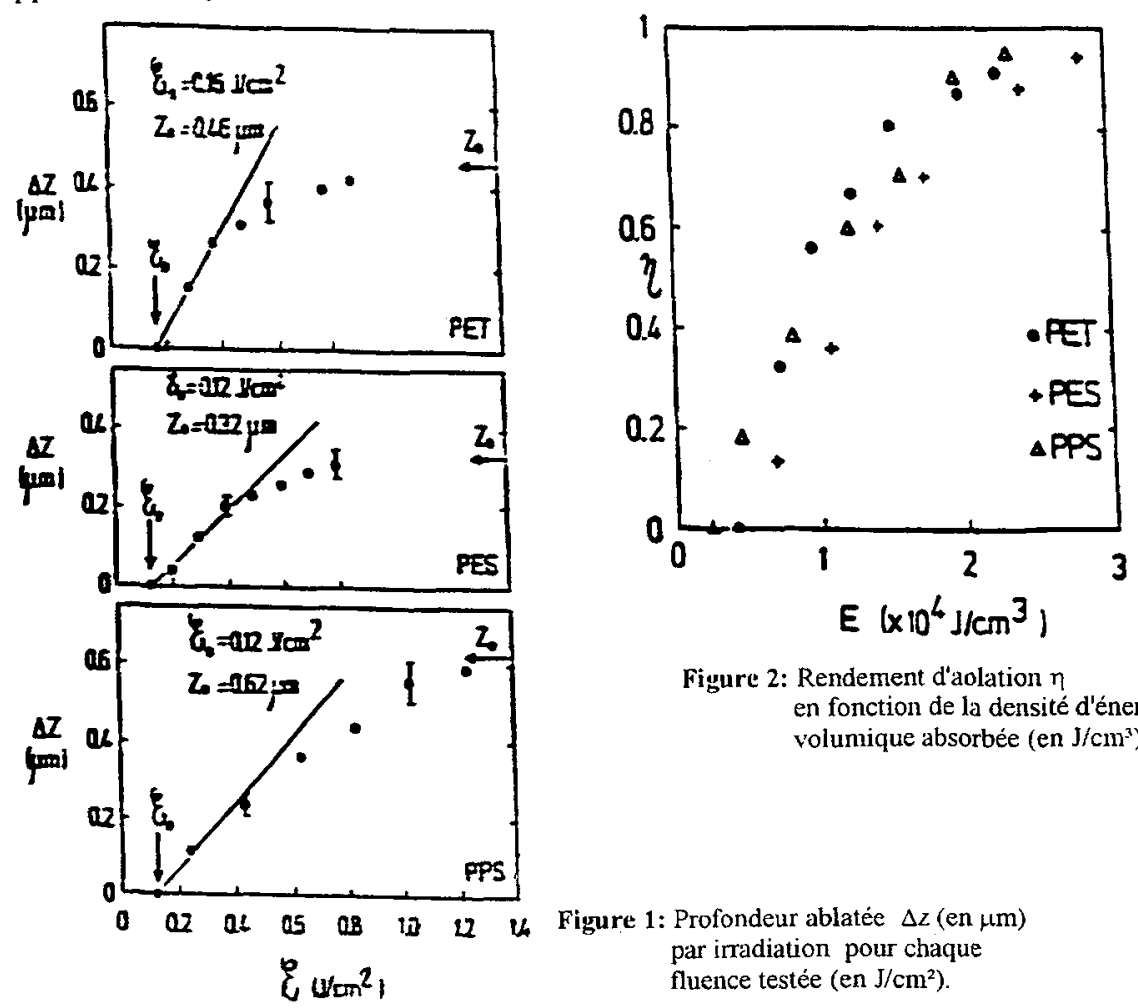

Figure 2: Rendement d'aolation $\eta$ en fonction de la densité d'énergie volumique absorbée (en $\mathrm{J} / \mathrm{cm}^{3}$ ).

Figure 1: Profondeur ablatée $\Delta z$ (en $\mu \mathrm{m}$ ) par irradiation pour chaque fluence testée $\left(\mathrm{en} \mathrm{J} / \mathrm{cm}^{2}\right)$. 
En tant que telles, ces quantités peuvent être raisonnablement considérées comme représentatives des matériaux testés. Elles demeurent pourtant purement descriptives et n'apportent aucune information utile en relation d'une part, avec la structure des polymères et d'autre part, avec leur comportement vis-à-vis de la source de lumière qui a induit l'ablation. Intrinsèquement, ces résultats ne sont aussi guère différents dans leur nature de ceux publiés par ailleurs pour d'autres polymères.

\section{INTERPRETATION}

\section{Le seuil d'ablation}

L'énergie disponible par $\mathrm{cm}^{2}$ de surface irradiée s'exprime comme $E_{0}=(1-R) E_{0}$. Du fait du processus d'absorption de cette énergie, au travers de l'interaction photon-électron et de la probabilité d'absorption de chacun des photons disponibles par un des électrons de valence, le rayonnement tend à pénétrer le matériau sur une certaine profondeur. Pour autant que ce matériau soit homogène, cette pénétration peut être régie par une loi de type Lambert, donnant l'intensité disponible à la profondeur $z$ sous la forme: $E(z)=E_{0} \exp (-K z)$, où $K$ est un coefficient d'absorption phénoménologique en charge de la probabilité d'absorption. On peut alors montrer aisément que $90 \%$ de cette intensité sont absorbés sur une profondeur de pénétration définie par $z_{y(1}=2,3 / \mathrm{K}$. Pour un rayonnement monochromatique, $\mathrm{K}$ est défini de façon univoque: il dépend de l'énergie propre du photon ( $h v=5,0 \mathrm{eV}$ à $248 \mathrm{~nm}$ ) et de la structure électronique du matériau et ne dépend pas de $\mathbf{E}_{0}$, c'est-à-dire du nombre de photons incidents et donc de la fluence $E$ : il en est de même de la profondeur de pénétration $z_{90}$. Pour un matériau donné et à une longueur d'onde donnée, la pénétration du rayonnement est la même quelle que soit la fluence.

Par ailleurs, on peut admettre que, d'une part le volume de matière émise lors d'une l'ablation appartient à cette partie du matériau qui est pénétrée par le rayonnement et, d'autre part ne peut l'excéder quelle que soit la fluence. Or on observe, Fig. 1, que, lorsqu'on accroît la fluence au delà du seuil, la profondeur ablatée par irradiation tend systématiquement vers une valeur asymptotique maximum $z_{0}$. Il semble donc logique d'évaluer, en toute fluence, la profondeur de pénétration du rayonnement comme $z_{0}$. Les graphes de la Fig. 1 peuvent alors être modifiés en rapportant, en abscisse, l'énergie absorbée au volume pénétré, soit $\mathrm{E} / \mathrm{z}_{0} \mathrm{en} \mathrm{J} / \mathrm{cm}^{3}$, et en ordonnée, la fraction émise du volume pénétré ou rendement d'ablation $\eta=S \times \Delta z / S \times z_{17}=$ $\Delta z / z_{b}$, où $S$ est la surface irradiée. Ces nouveaux graphes sont représentés Fig. 2.

Le seuil d'ablation s'exprime ici en $\mathrm{J} / \mathrm{cm}^{3}$. Les trois valeurs obtenues sont remarquables en ce qu'elles sont d'abord en proportion exacte de la taille des monomères correspondants (population électronique). Elles permettent aussi d'évaluer la densité de population des électrons excités au seuil d'ablation et, par suite, la distance moyenne entre ces électrons.

\begin{tabular}{|c|c|c|c|}
\hline & PET & PES & PPS \\
\hline $\begin{array}{l}\text { * population électronique } \\
\text { du monomère }\end{array}$ & 72 & 80 & 34 \\
\hline * seuil d'ablation $\left(\mathrm{kJ} / \mathrm{cm}^{3}\right)$ & 3,24 & 3,41 & 1,78 \\
\hline électrons excités au seuil (nm) & 0,62 & 0,62 & 0,77 \\
\hline
\end{tabular}


Ces trois polymères ont leur seuil d'absorption optique entre $3,5 \mathrm{eV}(350 \mathrm{~nm})$ et $4,2 \mathrm{eV}$ $(300 \mathrm{~nm})$. Les seuls électrons de valence susceptibles d'interagir avec des photons de $5 \mathrm{eV}$ occupent donc une gamme d'énergie d'environ $1 \mathrm{eV}$ au sommet de la bande de valence de ces monomères. Un nombre croissant d'états électroniques de cette gamme qui se trouve être progressivement sollicité à mesure que la fluence est accrue jusqu'au voisinage du seuil. Etant donné les populations électroniques respectives et sans préjuger d'un calcul de la distribution des états de valence, on peut estimer que la bande de valence du PPS est très probablement nettement plus étroite que celle du PES ou du PET ce qui expliquerait la corrélation entre les valeurs du seuil (en $\mathrm{J} / \mathrm{cm}^{3}$ ) et la taille des monomères. Indépendamment, le nuage électronique généré par l'absorption optique devenant plus dense dans le volume pénétré par le rayonnement, la distance moyenne entre électrons excités décroît pour atteindre, au voisinage du seuil, des valeurs qui semblent suffisamment faibles pour permettre la mise en œuvre d'interactions coulombiennes répulsives entre ces électrons perturbant le processus d'absorption classique. Le seuil d'ablation (exprimé en $\mathrm{J} / \mathrm{cm}^{3}$ ) représente donc l'énergie optique maximum que peut absorber un monomère sans être déstabilisé, et cette limitation est très probablement la conséquence de l'émergence d'un plasma électronique saturé: le seuil d'ablation représenterait ainsi une mesure de la "capacité optique" du matériau.

\section{Le régime d'ablation}

Le volume émis de matière croît d'abord linéairement avec la fluence juste au dessus du seuil d'ablation. Cet accroissement est, respectivement, de : 0, 12 (PET), 0,07 (PES) et 0,09 (PPS) $\mathrm{cm}^{3} / \mathrm{kJ}$. En associant un volume de $0,03 \mathrm{~nm}^{3}$ à chaque atome, ces quantités correspondent, en moyenne pour les trois polymères et de façon très caractéristique, à 2 ou 3 atomes émis par photon absorbé en excès du flux de photons correspondant au seuil. Sans entrer dans le détail d'un modèle dynamique de l'ablation qui est présenté in extenso ailleurs, il apparaît très clairement que la structure du polymère est conduite ainsi à éclater en débris contenant en moyenne plus de un atome, alors que, dans un régime d'interaction classique (état solide), un seul photon n'est sensé déstabiliser qu'une seule liaison interatomique en excitant un seul électron de valence. Par ailleurs, si on considère que le plasma électronique a atteint alors une taille critique qui lui interdit de croître davantage, les photons incidents qui sont en excès du flux correspondant au seuil ne pourraient plus interagir qu'avec ce plasma pour le maintenir en l'état, permettant à l'émission de matière de se développer

\section{CONCLUSION}

Les profils d'ablation d'une famille de trois polymères ont été déterminés. L'interprétation qui en est proposée a été concentrée sur le sens physique du seuil d'ablation. En particulier, on montre que ce seuil est proportionné à la taille des monomères et pourrait être la conséquence de la création d'un plasma électronique dense dans le volume du matériau irradié.

\section{Remerciements}

Ce travail a été réalisé dans le cadre d'un contrat de recherche octroyé par le Ministère de la Région Wallonne que les auteurs remercient.

\section{Références}

1. B. Braren, R. Srinivasan, J. Vac. Sci. Technol. B 3(3), 913 (1985)

2. S. Lazare, J. Lopez, F. Weisbuch, Appl. Phys. A 69 (Suppl.) S1-S6 (1999)

3. B. Luk'yanchuk et al., in Excimer Lasers, 59-77 (L.D.Laude ed., 1994 Kluwer Acad. Publ.) 